\title{
Transcriptomic profiling of two Pak Choi varieties with contrasting anthocyanin contents provides an insight into structural and regulatory genes in anthocyanin biosynthetic pathway
}

\author{
Lu Zhang $^{1 *}$ (D), Bin Xu' ${ }^{2}$, Tao Wu ${ }^{1}$, Yanfang Yang ${ }^{3}$, Lianxue Fan ${ }^{1}$, Muxuan Wen ${ }^{1}$ and Jiaxin Sui ${ }^{1}$
}

\begin{abstract}
Background: The accumulation of anthocyanin in horticultural crops not only improves their stress tolerances but also their nutritional values. Many key regulatory and structural genes in anthocyanin biosynthesis have been identified in model plants, but limited information is available for non-model plant species featured with colored leaves. In this study, two Pak Choi varieties with green or purple leaves were selected to analyze the anthocyanin biosynthesis through RNA-Seq.

Results: A total of 2475 unigenes were differentially expressed between these tested varieties, including 1303 downregulated and 1172 up-regulated genes in the purple-leafed one. The reliability of the RNA-Seq was further confirmed by using real-time quantitative PCR. Kyoto Encyclopedia of Genes and Genomes enrichment analysis of the differentially expressed genes revealed 'flavonoid biosynthesis' was the only enriched pathway in the purple-leafed variety: In the pathway of phenylpropanoid metabolism, Bra017210, Bra039777, and Bra021637 were expressed at higher levels in the purple-leafed variety; among the early anthocyanin biosynthetic genes, Bra037747 transcripts were only detected in the purple-leafed variety but not in the green-leafed one; among the late anthocyanin biosynthetic genes, Bra027457, Bra013652, Bra019350, Bra003021, Bra035004, and Bra038445 were all up-regulated in purple-leafed variety; and genes encoding anthocyanin-related transcription factors, such as Bra016164, and genes encoding anthocyanin transportation, such as GST F12, were also identified as up-regulated ones in the purple-leafed variety.

Conclusions: The current result provided a valuable insight into the anthocyanin accumulation in the purple-leafed variety of Pak Choi and a bioinformatic resource for further functional identification of key allelic genes determining the difference of anthocyanin content between Pak Choi varieties.
\end{abstract}

Keywords: Anthocyanin, Brassica campestris L. ssp. chinensis L. Makino, Transcriptome, RNA-Seq

\footnotetext{
* Correspondence: caszhanglu@hotmail.com

${ }^{1}$ College of Horticulture, Northeast Agricultural University, 59 Mucai street,

150030 Harbin, People's Republic of China

Full list of author information is available at the end of the article
} 


\section{Background}

Most autotrophic higher plants in temperate regions are featured with leaf coloration during the progression of leaf senescence in autumn or under stress conditions mainly due to the accumulation of anthocyanins [1]. However, there are plants having inherent colored leaves (e.g. red or purple) during their entire life cycles. Horticultural crops with high anthocyanin contents are considered valuable for the health-promoting effect of anthocyanins [2]. The accumulation of anthocyanin is also proposed as an important strategy for plant tolerance against abiotic or biotic stresses at least partially through its photoprotective function against excessive sunlight and its antioxidant function for mitigating the signal transduction of and the damage effect of reactive oxygen species [3].

The anthocyanin biosynthesis pathway has been intensively studied in model plant Arabidopsis [4], which is initiated from branches of the flavonoid pathway: starting from the conversion of phenylalanine to cinnamic acid by phenylalanine ammonia-lyase (PAL), and then sequentially catalyzed by cinnamate 4-hydroxylase $(\mathrm{C} 4 \mathrm{H})$, 4-coumaroyl:CoA-ligase (4CL), chalcone synthase (CHS), chalcone isomerase (CHI) to form naringenin. Naringenin can be further catalyzed by flavanone 3-hydroxylase (F3H), dihydroflavonol 4-reductase (DFR), and anthocyanidin synthase (ANS/LDOX) to form pelargonidin [4-6], or catalyzed by two other enzymes flavonoid 3'-hydroxylase $\left(\mathrm{F} 3{ }^{\prime} \mathrm{H}\right)$ or flavonoid $3{ }^{\prime} 5^{\prime}$-hydroxylase $\left(\mathrm{F} 3{ }^{\prime} 5^{\prime} \mathrm{H}\right)$ to hydroxylate the B-ring of dihydrokaempferol (DHK) into dihydroquercetin or dihydromyricetin that eventually forms cyanidin or delphinidin, respectively. In another branch, naringenin can be directly catalyzed by DFR and ANS to form apigenidin. Finally, these unstable anthocyanidins are glycosylated immediately to form anthocyanins [7]. Despite of the fact that this biosynthetic pathway is relatively conserved and finely characterized in model plants, deep insights are just recently gained into its upstream regulatory mechanism.

The anthocyanin biosynthesis pathway genes were regulated by transcription factors, such as the conserved MBW complex composed of MYB, basic HelixLoop-Helix (bHLH) and WD40 subunit proteins in higher plants [8-10]. This MBW complex played essential roles in the synergistic regulation of anthocyanin accumulation through inhibiting or activating the expression of related genes [11-14], and this complex per se is repressed by MYBL2 and JAZ family proteins [15]. Furthermore, this MYBL2-JAZ repressor complex is sequestered by DELLA proteins [15]. This JAZDELLA-MYBL2 module upstream of the MBW complex together mediated abiotic stress-caused anthocyanin accumulation in Arabidopsis [15]. While the transferability of this knowledge into non-model plants needs to be further tested, it is of particular interest to understand the regulatory mechanism of persistent accumulation of anthocyanin at high levels in horticultural plants where high contents of anthocyanins are often desirable.

Pak Choi (Brassica Campestris L. ssp. chinensis L. Makino), one of the most consumed vegetables in China and phylogenetically close to Arabidopsis contains different varieties with highly varied levels of anthocyanin [16, 17], which makes Pak Choi an ideal plant system for studying the mechanism underlying persistence accumulation of anthocyanin. The objective of this study was to identify and characterize key genes and regulating elements involved in leaf anthocyanin accumulation in Pak Choi through RNA-Seq with Brassica rapa L. spp. pekinensis as reference.

\section{Methods}

\section{Plant materials}

Two varieties of Pak Choi with different leaf colors were selected in this study: 'Jingguan' with green leaves (abbreviated as ' $G$ ' in this study), and 'Zizuan' with purple leaves (abbreviated as 'P'). Seeds of 'G' were from Beijing Vegetable Research Center, Beijing Academy of Agriculture and Forestry Sciences, and those of 'P' were from Beijing Dongsheng Seed Company. The seeds were sown in pots at $25{ }^{\circ} \mathrm{C}$ in July 2015 in a modern greenhouse located in Chinese Academy of Forestry Sciences, Beijing, China. When the seedlings had five leaves in total, the third leaf from the bottom was sampled and stored at $-80{ }^{\circ} \mathrm{C}$ before further processing. Six biological replicates were applied for anthocyanin content measurements, and three replicates for the RNA-Seq analysis.

\section{Analysis of total anthocyanin}

Fresh leaf tissue $(0.2 \mathrm{~g})$ was collected for the measurement of anthocyanin content. In brief, anthocyanins were extracted in $10 \mathrm{ml}$ acidified methanol $\left(99 \mathrm{CH}_{3} \mathrm{OH}: 1 \mathrm{HCl}, \mathrm{v} / \mathrm{v}\right)$ at $4{ }^{\circ} \mathrm{C}$ for $24 \mathrm{~h}$ in the dark, clarified by centrifugation at $12,000 \times g$ for $2 \mathrm{~min}$, and the absorbance of supernatants was determined using a UV-visible spectrophotometer (UV-2550, Shimadzu, Japan) from optical density (OD) at 530 and $600 \mathrm{~nm}$. Anthocyanin concentrations were expressed as U.g ${ }^{-1}$, where U was calculated as (OD530-OD600)/0.1 [18].

\section{RNA extraction and library preparation for transcriptome analysis}

A total of $3 \mu \mathrm{g}$ RNA per sample was applied for the RNA sample preparations. Sequencing libraries were generated using NEBNext ${ }^{\oplus}$ Ultra $^{\text {Ts }}$ RNA Library Prep Kit for Illumina ${ }^{\circledR}$ (NEB, USA) according to manufacturer's instructions. By using poly-T oligo-attached magnetic 
beads, mRNA was purified from total RNA. Fragmentation was operated using divalent cations under elevated temperature in NEBNext First Strand Synthesis Reaction Buffer (5X). First strand cDNA was synthesized using random hexamer primer and M-MuLV Reverse Transcriptase (RNase $\mathrm{H}^{-}$). Second strand cDNA synthesis was then performed using DNA Polymerase I and RNase $\mathrm{H}$. Remaining overhangs were converted into blunt ends via exonuclease/polymerase activities. After adenylation of 3' ends of DNA fragments, NEBNext Adaptor with hairpin loop structure were ligated to be ready for hybridization. To select cDNA fragments of $150 \sim 200 \mathrm{bp}$ in length, the library fragments were purified with AMPure XP system (Beckman Coulter, Beverly, USA). Then $3 \mu$ l USER Enzyme (NEB, USA) was used with size-selected, adaptor-ligated cDNA at $37{ }^{\circ} \mathrm{C}$ for $15 \mathrm{~min}$ followed by $5 \mathrm{~min}$ at $95{ }^{\circ} \mathrm{C}$ before PCR. Then PCR was performed with Phusion High-Fidelity DNA polymerase, Universal PCR primers and Index (X) Primer. In the end, PCR products were purified (AMPure XP system) and the assessment of library quality was conducted on the Agilent Bioanalyzer 2100 system. The library preparations were sequenced on an Illumina Hiseq 2500 platform and 125 bp paired-end reads were generated.

\section{Analysis of Illumina sequencing results}

Clean data (clean reads) were obtained by deleting reads containing poly- $\mathrm{N}$, reads containing adapters, and low quality reads from raw data. At the same time, Q20, Q30, GC-content of the clean data were calculated. Based on clean data of high quality, the downstream analyses were conducted.

\section{Quantification of gene expression levels and differential expression analysis}

Reference genome and gene model annotation files were downloaded from Brassica database website directly [http://brassicadb.org/brad/] [19]. Clean data were mapped back onto the reference genome using TopHat v2.0.12. The HTSeq v0.6.1 software was used to count the reads numbers mapped onto each gene. And the expected number of Fragments per Kilobase of transcript sequence per Millions base pairs sequenced (FPKM) of each gene was calculated based on the length of the gene and reads count mapped to this gene. Differential expression analysis of two varieties (three biological replicates per variety) was performed using DEseq program [20]. Genes with an adjusted $P$-value $<0.05$ found by DESeq were considered as differentially expressed. Gene Ontology (GO) enrichment analysis of the differentially expressed genes (DEGs) was conducted by the GO seq R packages based Wallenius non-central hyper-geometric distribution [21], which can adjust for gene length bias in DEGs. KOBAS 2.0 [22] was used to test the statistical enrichment of DEGs in Kyoto Encyclopedia of Genes and Genomes (KEGG) pathways.

\section{Real-time quantitative RT-PCR (RT-qPCR) assay}

The expression patterns of nine genes involved in the anthocyanin pathway (Gene ID: Bra013652, Bra019350, Bra027457, Bra037747, Bra017520, Bra017523, Bra026967, Bra016164, and, Bra032635) were analyzed using qRT-PCR. cDNA was synthesized using ReverseTra Ace qPCR RT Kit (Toyobo, Japan) according to the manufacturer's recommendation. The reverse transcription reaction system contained $2 \mu \mathrm{L} 5 \times \mathrm{RT}$ buffer, $0.5 \mu \mathrm{L}$ primer mix, $0.5 \mu \mathrm{L}$ RT enzyme mix, $2 \mu \mathrm{L}$ RNA

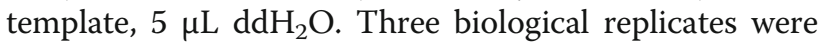
applied for each gene expression analysis. Gene-specific primers were designed according to the reference unigene sequence using the online tool [GenScript Real-time PCR (TaqMan) Primer Design, https://www.genscript.com/sslbin/app/primer]. The cDNA diluted to $100 \mathrm{ng} / \mu \mathrm{L}$ was used for qPCR assay with each gene-specific primers and SYBR $^{\circ}$ Green Real time PCR Master Mix (Toyobo, Japan) on the Bio-Rad iQ5 real time system. Reactions were performed at $96^{\circ} \mathrm{C}$ for $1 \mathrm{~min}, 40$ cycles of $95^{\circ} \mathrm{C}$ for $15 \mathrm{~s}, 60^{\circ}$ $\mathrm{C}$ for $15 \mathrm{~s}$ and $72{ }^{\circ} \mathrm{C}$ for $45 \mathrm{~s}$. All primers for RT-qPCR are listed (Additional file 1: Table S1).

\section{Results}

Quantification of anthocyanin contents of two Pak Choi varieties

Two Pak Choi varieties were used in this study with contrasting colors that the purple variety (hereafter abbreviated as ' $\mathrm{P}$ ') had $11.703 \mathrm{U} \cdot \mathrm{g}^{-1}$ of anthocyanins while the green one (hereafter abbreviated as ' $G$ ') only had $0.320 \mathrm{U} \cdot \mathrm{g}^{-1}$ (Table 1).

\section{RNA-sequencing of two Pak Choi varieties with different leaf colors}

Leaves at the same growth stage were sampled from these two varieties and their transcriptomes were profiled using Illumina sequencing. In total, 172,380,454 and $168,368,500$ raw reads were generated from the ' $G$ ' and 'P' libraries, respectively (Table 2). To ensure the quality of the libraries, adaptor reads, ambiguous reads and low-quality reads were removed (Fig. 1). Finally,

Table 1 Anthocyanin contents of two Pak Choi varieties with different leaf colors

\begin{tabular}{lc}
\hline Variety & Anthocyanin contents $\left(\mathrm{U} \mathrm{g}^{-1}\right.$ Fresh weight $)$ \\
\hline Green-leafed variety ' $G$ ' & $0.320 \pm 0.054 \mathrm{~b}$ \\
Purple-leafed variety ' $P$ ' & $11.703 \pm 1.360 \mathrm{a}$ \\
\hline
\end{tabular}

Values are Mean \pm SD of 6 replications. Values followed by different letters indicate significant difference at $P<0.05$ 
Table 2 Summary of sequences analysis and RNA-Seq data

\begin{tabular}{lll}
\hline Sample name & Green-leafed variety 'G' & Purple-leafed variety 'P' \\
\hline Raw reads & $172,380,454$ & $168,368,500$ \\
Clean reads & $165,289,336$ & $161,562,282$ \\
Clean bases & $24.79 G$ & $24.24 G$ \\
Error rate (\%) & 0.01 & 0.01 \\
Q20 (\%) & 97.97 & 98.01 \\
Q30 (\%) & 94.95 & 94.99 \\
GC content (\%) & 48.03 & 47.91 \\
Total mapped & $111,825,953(67.63 \%)$ & $112,860,042(69.89 \%)$ \\
Multiple mapped & $2,211,063(1.33 \%)$ & $2,111,248(1.33 \%)$ \\
Uniquely mapped & $109,614,890(66.30 \%)$ & $110,748,794(68.57 \%)$ \\
Reads map to '+' & $54,945,243(33.24 \%)$ & $55,565,180$ (34.40\%) \\
Reads map to '-' & $54,669,647$ (33.07\%) & $55,183,614(34.16 \%)$ \\
Non-splice reads & $63,472,079$ (38.40\%) & $65,056,741$ (40.27\%) \\
Splice reads & $46,142,811$ (27.90\%) & $45,692,053$ (28,29\%) \\
\hline Q20: The percentage of bases with a Phred value >20 \\
Q30: The percentage of bases with a Phred value >30
\end{tabular}

$165,289,336$ and $161,562,282$ clean reads were obtained for ' $G$ ' and 'P', respectively (Table 2). A total of $67.63 \%$ reads in green-leafed variety and $69.89 \%$ reads in purple-leafed variety were mapped in the Brassica database (BRAD). On average, 66.3\% and 68.57\% mapped reads were uniquely mapped to the database (Table 2).

\section{Differentially expressed genes (DEGs) between the two Pak Choi varieties}

Normalized-FPKM (fragments per kilobase per million) were used to quantify the transcript levels. The FPKM of the two varieties ('G' and 'P') were almost the same (Fig. 2) for unbiased analysis of their transcript profiles. The DEGs were analyzed to identify candidate genes related to the synthesis of anthocyanins. DEGs (padj $<0.05$ ) were defined as genes that were significantly enriched or depleted in one variety relative to the other one. Between ' $G$ ' and 'P', a total of 2475 DEGs were identified, including 1303 down-regulated and 1172 upregulated DEGs in 'P' (Additional file 2: Table S2).

\section{Verification of RNA-Seq data by real-time quantitative RT- PCR}

To confirm the reliability of the RNA-Seq data, the relative expression levels of nine DEGs were examined by real-time quantitative PCR (RT-qPCR). Among the tested genes, one encoded a member of the oxidoreductase family protein, four transcription factor genes, and four anthocyanin biosynthetic enzymes. Consistent with the RNA-Seq data, relative expression levels of all selected genes were higher in the purple-leafed variety than in the green-leafed variety. This result further confirmed the reliability of the RNA-Seq data (Table 3).
Classification of Raw Reads (G_1)

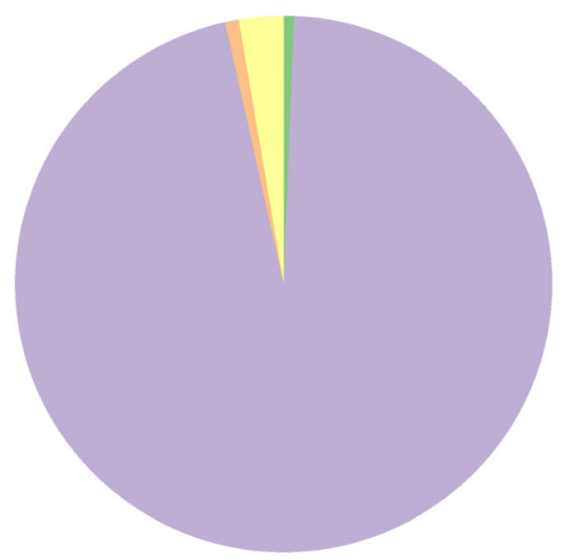

Clean Reads (28274538, 95.88\%) Containing N (790924, 2.68\%)

Low Quality (243186, 0.82\%)

Adapter Related (180906, 0.61\%)
Classification of Raw Reads ( $\left.P_{-} 1\right)$

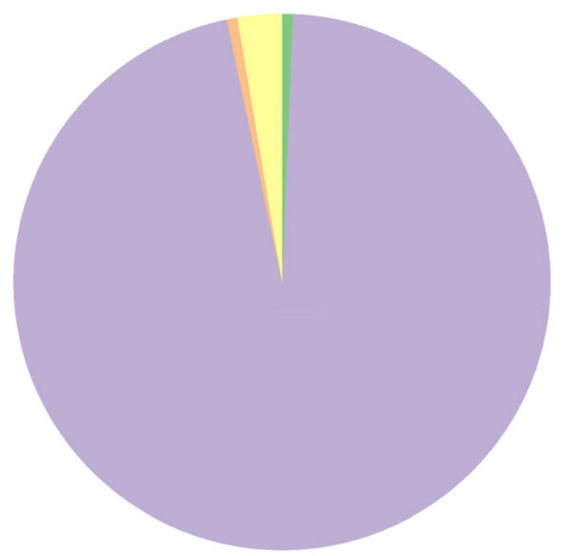

Clean Reads (24450600, 96.04\%) Containing N (678371, 2.66\%)

Low Quality (164094, 0.64\%)

Adapter Related (165731, 0.65\%)

Fig. 1 The quality of raw reads of green-leafed variety ('G') and purple-leafed variety ('P') of Pak Choi using RNA-Seq 


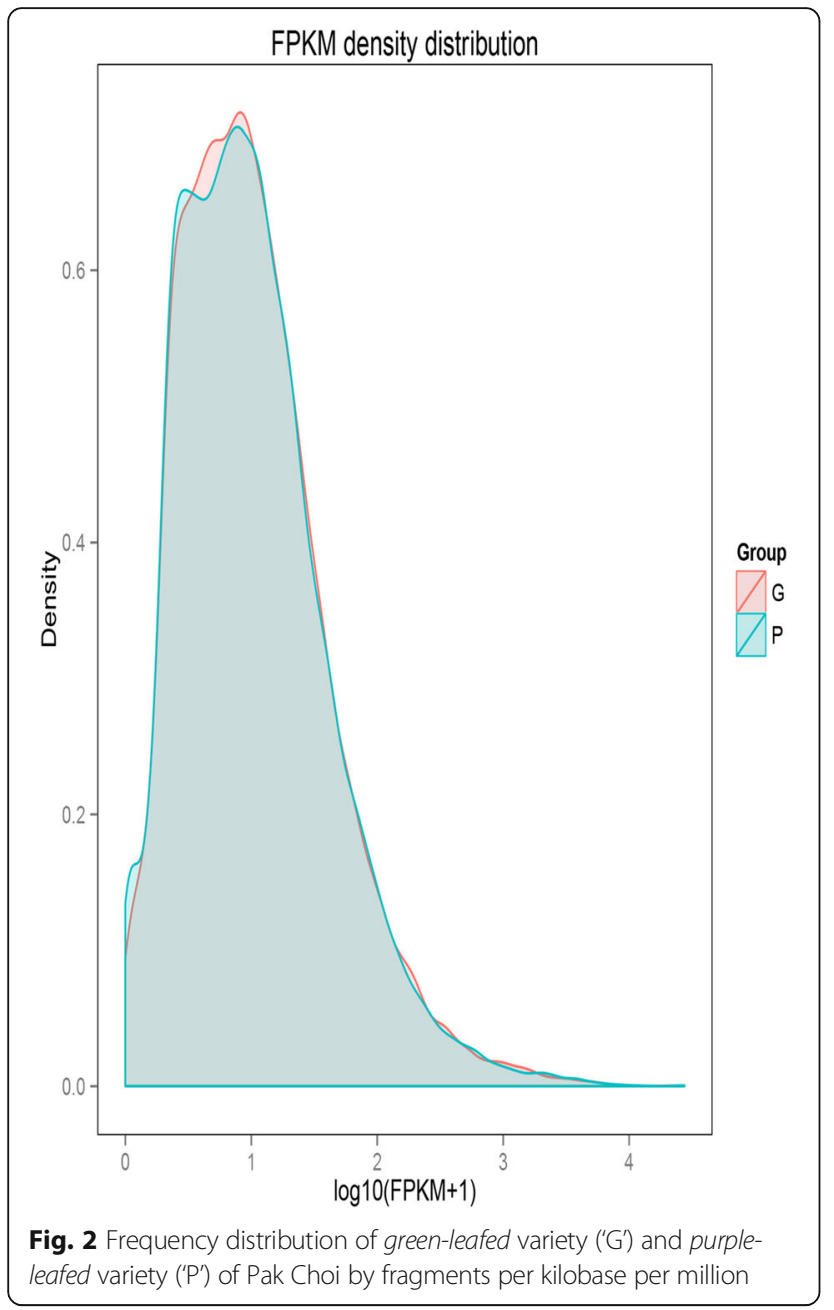

\section{Functional classification of DEGs}

GO classifications were conducted in order to predict DEGs' functions. GO enrichment analysis (corrected $p$-value $<0.5)$ of the up-regulated DEGs in the ' $\mathrm{P}$ ' suggested their variety-specific functions. In the category of biological processes, the highly enriched DEGs in ' $\mathrm{P}$ ' included those involved in 'carboxylic acid metabolic process', 'oxoacid metabolic process', 'organic acid metabolic process', 'organic acid biosynthetic process', 'carboxylic acid biosynthetic process' and 'RNA-dependent DNA replication'. In the molecular function category, the 'oxidoreductase activity' and 'oxidoreductase activity, acting on the $\mathrm{CH}-\mathrm{OH}$ group of donors, NAD or NADP as acceptor' were the mostly highly enriched (Fig. 3). GO analysis (corrected $p$-value $<0.2)$ was also conducted for the downregulated DEGs in 'P' (Fig. 4). In the category of biological processes, 'single-organism process' and 'single-organism cellular process' were the mostly highly enriched ones; and in the category of molecular function, the top enriched terms were 'anion binding', and 'carbohydrate derivative binding', (Fig. 4).

The result of KEGG pathway enrichment analysis for DEGs showed that the only enriched pathway of DEGs (corrected- $P$ value is almost 0.05 ) was 'flavonoid biosynthesis' [KEGGmap00941 (http://www.genome.jp/ dbget-bin/www_bget?map00941)] (Fig. 5), and there were 11 DEGs between ' $P$ ' and 'G' involved in this pathway (Fig. 6). Most of these genes were upregulated in purple-leafed variety. For examples, the biosynthetic genes of anthocyanins, Bra027457, Bra013652, Bra019350, Bra003021, Bra035004, and Bra038445 were all up-regulated in 'P' (Table 4). In the phenylpropanoid metabolism, Bra017210, Bra039777, and Bra021637 were expressed at a higher level in 'P' than those in 'G'. However, Bra006985 had higher expression in ' $G$ ' than those in ' $P$ '. For biosynthetic genes in upstream of the anthocyanin biosynthesis pathway, Bra037747 was expressed in 'P' rather than in ' $G$ ', yet Bra029212 was expressed at a lower level in 'P' than those in ' $G$ '. Genes involved in anthocyanin transport

Table 3 The expression patterns of selected genes using real-time quantitative RT-PCR and RNA-Seq

\begin{tabular}{|c|c|c|c|c|c|}
\hline \multirow[t]{2}{*}{ Gene } & \multicolumn{2}{|l|}{ RT-PCR } & \multicolumn{2}{|c|}{ RNA-Seq (FPKM) } & \multirow[t]{2}{*}{ Gene annotation } \\
\hline & 'G' & 'P' & 'G' & 'P' & \\
\hline Bra013652 & $0.88 \pm 0.16 b$ & $179.31 \pm 87.33 \mathrm{a}$ & 79.26 & 11936.77 & Leucoanthocyanidin dioxygenase \\
\hline Bra019350 & $1.00 \pm 0.05 b$ & $2.72 \pm 0.41 \mathrm{a}$ & 23.62 & 4969.47 & Leucoanthocyanidin dioxygenase \\
\hline Bra027457 & $1.35 \pm 0.47 b$ & $23.61 \pm 2.38 \mathrm{a}$ & 67.91 & 15507.10 & Dihydroflavonol-4-reductase \\
\hline Bra037747 & $1.13 \pm 0.11 b$ & $3.08 \pm 0.47 a$ & 0 & 26.11 & Flavonol synthase/flavanone 3-hydroxylase \\
\hline Bra017520 & $1.18 \pm 0.31 b$ & $14.76 \pm 3.48 \mathrm{a}$ & 0.94 & 21.80 & Basic helix-loop-helix (bHLH) family protein \\
\hline Bra017523 & $1.11 \pm 0.14 b$ & $28.85 \pm 0.80 a$ & 16.31 & 540.00 & Basic helix-loop-helix (bHLH) family protein \\
\hline Bra026967 & $1.12 \pm 0.14 b$ & $8.88 \pm 1.45 a$ & 0 & 12.00 & oxidoreductase family protein \\
\hline Bra016164 & $0.93 \pm 0.11 b$ & $193.23 \pm 8.24 a$ & 5.87 & 865.19 & MYB08 Myb-related protein 308 \\
\hline Bra032635 & $0.01 \pm 0.00 \mathrm{~b}$ & $1.04 \pm 0.03 \mathrm{a}$ & 2.84 & 373.93 & ETC1 (ENHANCER OF TRY AND CPC 1); DNA binding / transcription factor \\
\hline
\end{tabular}

The relative quantitation of gene expression was conducted via the $2^{-\Delta \Delta C t}$ method, with actin as an endogenous reference. Data from three biological replicates were used to calculate the mean and standard deviation in DPS based on Student's $t$-test. Values followed by different letters indicate significant difference at $P<$ 0.05. ' $G$ ': Green-leafed variety; ' $P$ ': Purple-leafed variety 
The Most Enriched GO Terms P vs G

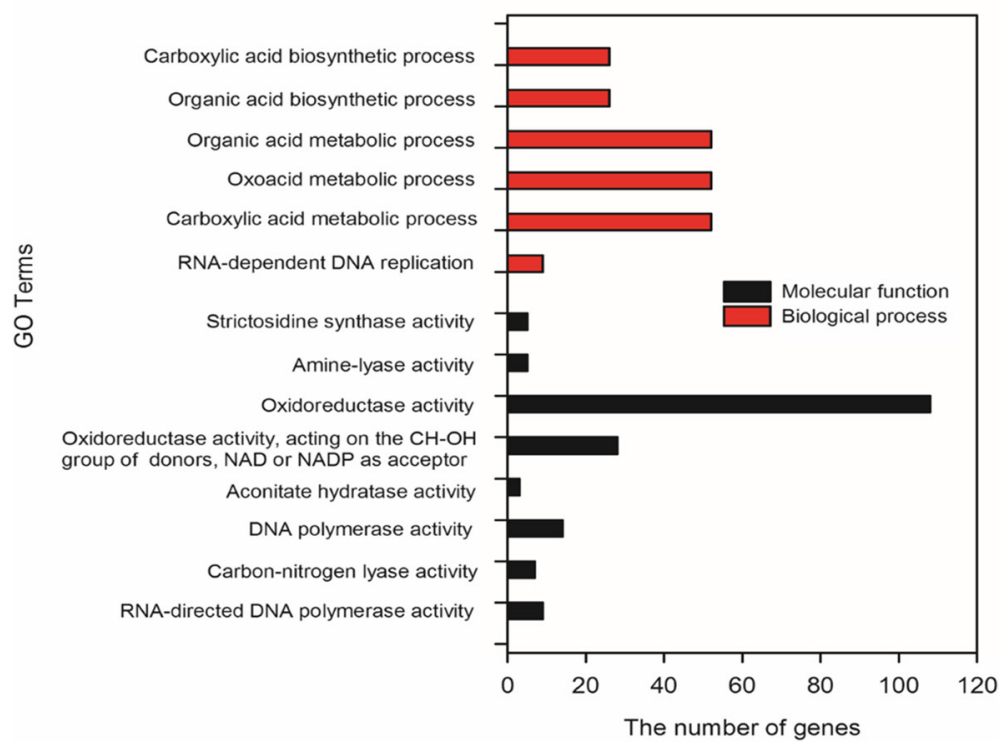

Fig. 3 Go enrichment of up-regulated genes (corrected $p$-value $<0.5$ ) in leaves of purple-leafed variety ('P') compared with those of green-leafed variety ('G') of Pak Choi

were also identified, and the pair of allelic genes (Bra008570 and Bra023602) encoded Glutathione Stransferase $F 12$ were both significantly up-regulated in the purple-leafed variety (Table 4).

Transcription factors play essential roles in the transcriptional regulation of structural genes in anthocyanin biosynthesis. Therefore candidate transcription factors potentially involved in anthocyanin biosynthesis were further pinpointed in the RNA-Seq analysis. It was found that Bra036145 was suppressed, while Bra037887, Bra016164 and Bra039283 were all upregulated in purple-leafed variety (Table 5). Yet, Bra011772 was expressed at significantly lower level in purple-leafed variety than green-leafed one. The

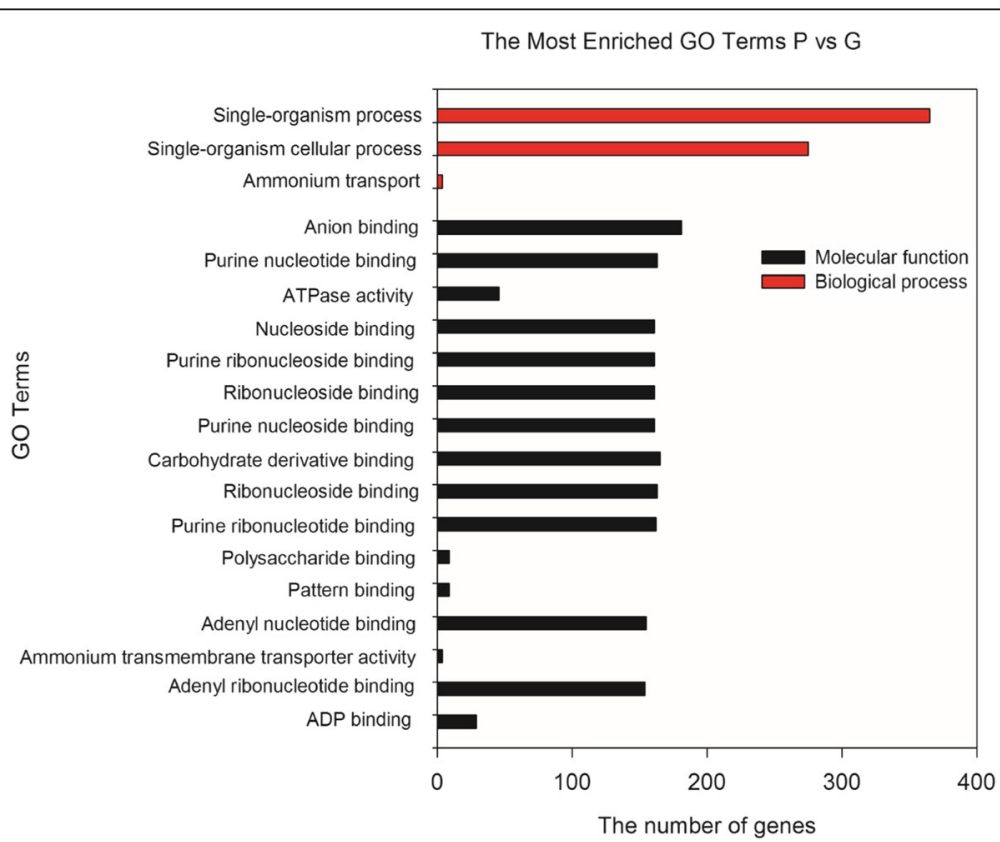

Fig. 4 Go enrichment of down-regulated genes (corrected $p$-value $<0.2$ ) in leaves purple-leafed variety (' $P$ ') compared with those of green-leafed variety ('G') of Pak Choi 


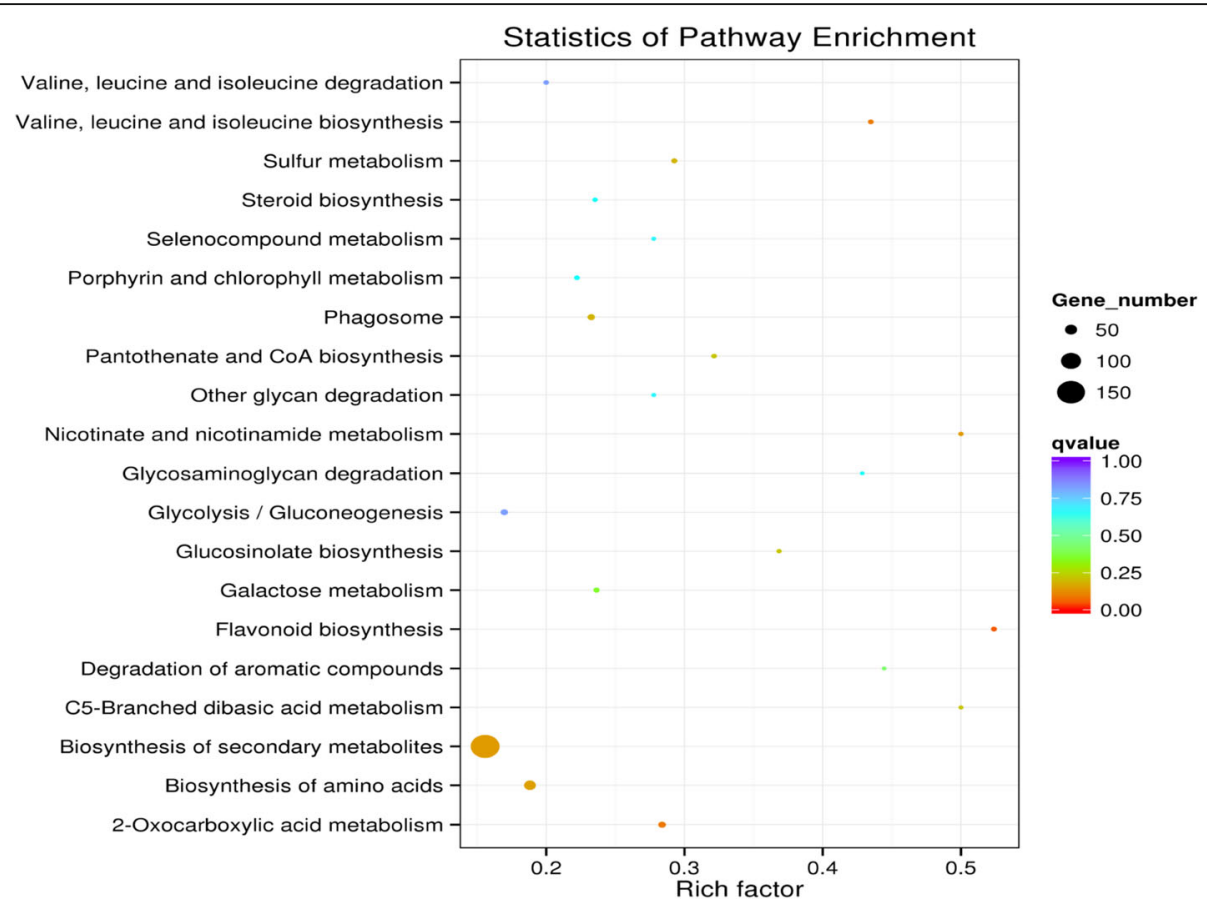

Fig. 5 KEGG enrichment of differentially expressed genes in leaves between green-leafed variety (' $G$ ') and purple-leafed variety ('P') of Pak Choi

predicated functions of these DEGs according to their Arabidopsis orthologs were shown in Table 5.

\section{Discussion}

The increased accumulation of anthocyanin in leafy vegetables improves their commercial and healthy values. Yet, there are limited studies on mechanisms underlying the anthocyanin biosynthesis in leafy vegetables [23]. In this study, transcriptomic analysis of the green-leafed and purple-leafed varieties of Pak Choi was conducted using RNA-Seq technology. The current results not only identified Pak Choi's vital structural genes involved in anthocyanin biosynthesis, but also revealed the potential regulatory mechanism involved in anthocyanin biosynthesis, transport and accumulation in the purple leafed variety.

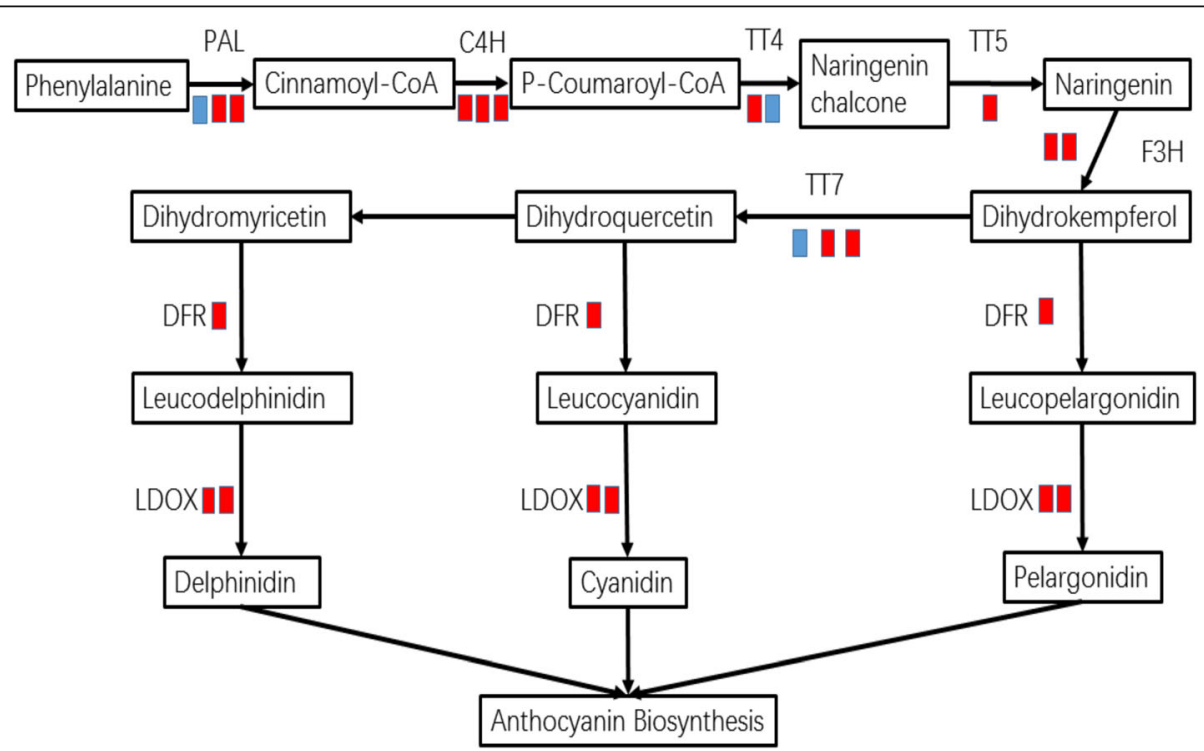

Fig. 6 Differential expressed genes predicted to be involved in the flavonoid biosynthesis pathway. Red block means the up-regulated genes and blue block means the down-regulated genes in purple-leafed variety (' $P$ ') compared with green-leafed variety (' $G$ ') of Pak Choi 
Table 4 The expression patterns of related genes for anthocyanin biosynthesis and transportation

\begin{tabular}{|c|c|c|c|c|}
\hline & \multirow[b]{2}{*}{ Gene ID } & \multirow[b]{2}{*}{ Gene annotation } & \multicolumn{2}{|l|}{ FPKM } \\
\hline & & & 'G' & 'P' \\
\hline \multirow[t]{5}{*}{ Biosynthetic genes in phenylpropanoid pathway } & Bra017210 & Phenylalanine ammonia-lyase 1 & 3566.60 & 8616.42 \\
\hline & Bra039777 & Phenylalanine ammonia-lyase 2 & 930.36 & 7009.32 \\
\hline & Bra006985 & Phenylalanine ammonia-lyase 2 & 3299.06 & 18.24 \\
\hline & Bra031266 & 4-coumarate-CoA ligase 2 & 216.25 & 624.71 \\
\hline & Bra021637 & Transcinnamate 4-monooxygenase & 939.76 & 4518.74 \\
\hline \multirow[t]{3}{*}{ Early Biosynthetic Genes } & Bra009101 & Probable chalcone-flavonone isomerase 3 & 1281.89 & 5917.44 \\
\hline & Bra037747 & Flavonol synthase/flavanone 3-hydroxylase & 0 & 26.11 \\
\hline & Bra029212 & Flavonol synthase 3 & 790.26 & 328.98 \\
\hline \multirow[t]{10}{*}{ Late Biosynthetic Genes } & Bra027457 & Dihydroflavonol-4-reductase & 67.91 & 15507.10 \\
\hline & Bra013652 & Leucoanthocyanidin dioxygenase & 79.26 & 11936.77 \\
\hline & Bra019350 & Leucoanthocyanidin dioxygenase & 23.6186 & 4969.47 \\
\hline & Bra035004 & Anthocyanidin 3-O-glucosyltransferase & 10.15 & 1736.71 \\
\hline & Bra003021 & Anthocyanidin 3-O-glucosyltransferase & 5.63 & 1011.59 \\
\hline & Bra038445 & UDP-glycosyltransferase $75 \mathrm{C} 1$ & 56.36 & 10314.18 \\
\hline & Bra011292 & Caffeic acid 3-O-methyltransferase 1 & 19.03 & 276.83 \\
\hline & Bra034600 & Caffeoyl-CoA O-methyltransferase 1 & 1080.65 & 6484.01 \\
\hline & Bra003009 & Quercetin 3-O-methyltransferase 1 & 8040.55 & 568.33 \\
\hline & Bra003707 & Caffeic acid 3-O-methyltransferase OS & 159.46 & 1.43 \\
\hline \multirow[t]{5}{*}{ Transport genes in anthocyanin pathway } & Bra009033 & Protein TRANSPARENT TESTA 12 & 22.96 & 3.61 \\
\hline & Bra016610 & ATPase 10, plasma membrane-type & 0.30 & 63.40 \\
\hline & Bra024452 & ATPase 1, plasma membrane-type & 6.84 & 199.33 \\
\hline & Bra008570 & Glutathione S-transferase F12 & 12.02 & 2134.23 \\
\hline & Bra023602 & Glutathione S-transferase F12 & 195.28 & 2391.95 \\
\hline
\end{tabular}

'G': Green-leafed variety; ' $P$ ': Purple-leafed variety

One objective of this study is to identify the structural genes involved in anthocyanin biosynthetic pathway. The current result confirmed the presence of all known structural genes in this pathway in Pak Choi, which result was in agreement with previous proposal that anthocyanin biosynthesis pathway and structural genes are relatively conserved across higher plants [24]. Yet, at the transcriptional level, great variations were found among these structural genes between ' $P$ ' and ' $G$ '. In the variety 'P', most genes in flavonoid pathway had significantly higher expression levels. For examples, our results showed that the expression of $\mathrm{Bra017210}$ and Bra039777, encoding for PAL1 and PAL2 respectively, were both higher in ' $P$ ' than in ' $G$ ', suggesting that the phenylpropanoid pathway which is upstream of anthocyanin biosynthesis was more active in the purple leafed variety $[25,26] .4 C L 2$ was another enzyme catalyzing the formation of hydroxycinnamic acid derivatives [27], and our study also revealed that the homologous gene of $4 C L 2$ was up-regulated in 'P'.

In the anthocyanin biosynthetic pathway, structural genes can be classified into two types: Early Biosynthetic

Table 5 Selected genes about transcription factor with altered expression

\begin{tabular}{|c|c|c|c|c|}
\hline & & & FPKM & \\
\hline Group & Gene ID & Gene Annotation & 'G' & 'P' \\
\hline \multirow[t]{5}{*}{ Transcription factor } & Bra016164 & MYB08 Myb-related protein 308 & 5.87 & 865.19 \\
\hline & Bra036145 & MYB12 Transcription factor MYB12 & 46.07 & 0 \\
\hline & Bra039283 & Transcription factor CPC & 0.98 & 42.77 \\
\hline & Bra037887 & Tா8 Transcription factor TT8 & 3.24 & 917.07 \\
\hline & Bra011772 & LBD39 & 101.15 & 19.33 \\
\hline
\end{tabular}

'G': Green-leafed variety; ' $P$ ': Purple-leafed variety 
Genes (EBGs) and Late Biosynthetic Genes (LBGs) [28]. The EBGs, including $\mathrm{CHS}, \mathrm{CHI}, \mathrm{F} 3 \mathrm{H}, \mathrm{F3} 3^{\prime} \mathrm{H}$, and $\mathrm{F3}^{\prime} \mathrm{5}^{\prime} \mathrm{H}$, catalyze the production of flavonols and other flavonoid compounds, while the LBGs, including DFR, ANS/ $L D O X$, and the UDP-glucose: flavonoid-3-O-glucosyltransferase (UFGTs), are specifically for anthocyanin biosynthesis [5, 29]. Our results showed that both early biosynthetic genes (Bra037747 and Bra029212) and late biosynthetic genes (Bra027457, Bra013652, Bra019350, Bra003021, Bra035004, and Bra038445) concurrent with the high anthocyanin content in 'P'. O-methyltransferase (OMT) plays important roles in catalyzing the methylation of anthocyanins [30]. And in 'P', the expression level of Caffeic acid 3-O-methyltransferase 1 and CaffeoylCoA O-methyltransferase 1 were both higher than those in 'G', while Quercetin 3-O-methyltransferase 1 and Caffeic acid 3-O-methyltransferase OS were lower in 'P' than in ' $G$ '. Together, these results confirmed that most structural genes were up-regulated for biosynthesis of anthocyanin which was in accordance with previous studies [31]. On the other side, exceptions did exist for the expression of the anthocyanin structural genes. For example, Bra029212 was expressed at a lower level in ' $\mathrm{P}$ ' than those in ' $G$ '. We reasoned that the possible reasons for such exceptions were that these genes either did not directly participate in the flavonoid biosynthesis pathway, or their expressions were suppressed due to the high-expression level and compensation effect of their redundant allelic genes. Nevertheless, these structural genes in anthocyanin biosynthesis were associated with the level of anthocyanin contents in 'P' and 'G'.

On the other hand, transcriptional regulation of the structural genes in anthocyanin biosynthesis pathway was an important regulatory strategy for anthocyanin formation and accumulation in Arabidopsis [28, 32]. These regulatory genes can be mainly classified into two groups: positive and negative regulatory genes. Unlike bHLH and WD proteins that may have broader and overlapping regulatory targets, MYB proteins are the key components providing specificity for the subsets of regulated genes [33]. It has been reported that three transcription factors (MYB11, MYB12, and MYB111) activate early biosynthetic genes of the anthocyanin biosynthetic pathway in Arabidopsis [34]. While in Pak Choi, there's only one ortholog of AtMYB111, but no ortholog of AtMYB11 and $A t M Y B 12$ was found either due to the absence of these genes in the Pak Choi genome or because of their extremely low expression level in both of these two Pak Choi varieties. The $b H L H$ gene family regulates anthocyanin biosynthesis through formation of MBW ternary complexes [35]. In Pak Choi, only one $b H L H$ transcriptional factor (TRANSPARENT TESTA8, TT8) was found that potentially involved in the regulation of anthocyanin biosynthetic genes. In Arabidopsis, TT8 not only regulates anthocyanidin production towards the synthesis of proanthocyanidins in seeds, but is also involved in the regulation of anthocyanin biosynthesis in vegetative tissues and cell cultures [35]. Our transcriptional result was also consistent with the functional role of TT8 in Pak Choi. There are several transcription factors including two R3-type single MYB proteins (MYBL2 and CPC) and three members of the LBD (LATERAL ORGAN BOUNDARIES DOMAIN) family that act as negative regulators of activities of WBM complexes decreasing anthocyanin biosynthesis in A. thaliana [5, 36]. Yet, the corresponding orthologs in Pak Choi (Bra016164 and Bra039283) showed higher expression levels in 'P' than in 'G', probably either due to the different but contradictory function of these two genes in Pak Choi or due to the feedback effect of the high anthocyanin content in ' $\mathrm{P}$ ' that in turn inhibited their expressions.

The flavonoid transporters also involved in the vacuolar transport of anthocyanins and proanthocyanidin precursors and might contribute to the accumulation of anthocyanin in plant as well [37-39]. In Arabidopsis, three genes, TT12, TT19 and AHA10, have been found to be related to the transport of anthocyanins. However, our results showed that Bra009033 was down regulated while genes encoding ATPase 10 and ATPase 1 were upregulated in purple-leafed variety. Glutathione Stransferases (GSTs) act as non-enzymatic carrier proteins enabling intracellular shuttling of endogenous compounds such as anthocyanin in plants. Previous gene knockout experiments revealed that GSTs were involved in anthocyanin transport [40] and the deposition of anthocyanin pigment into the vacuole [41]. The differential expressions of the GST encoding genes were found in this study, indicating a possible link between GST and the transportation of anthocyanin in Pak Choi as well.

\section{Conclusions}

We performed RNA-Seq for two varieties of Pak Choi with contrasting different anthocyanin contents. Nine structural genes in the anthocyanin biosynthetic pathway were up-regulated in the purple-leafed variety. Among them, the late biosynthetic genes including Bra027457, Bra013652, Bra019350, Bra003021, Bra035004, and Bra038445, probably played more pivotal roles in the biosynthetic process of anthocyanin. In addition, key transcription factor and transporter genes, such as Bra016164 and Bra009033, were also identified for the regulation of anthocyanin accumulation and transportation. The results in this study paved groundwork for further functional study on anthocyanin-related genes that will ultimately decipher the mechanism underlying persistently high anthocyanin contents in certain varieties of Pak Choi, and such knowledge shall also be useful for the other leafy horticultural crops. 


\section{Additional files}

Additional file 1: Table S1. The primers used for qRT -PCR. (DOCX $15 \mathrm{~kb}$ ) Additional file 2: Differentially expressed genes (DEGs) between the two Pak Choi varieties. (XLSX 256 kb)

\section{Abbreviations}

4CL: 4-coumaroyl:CoA-ligase; ANS/LDOX: Anthocyanidin synthase; bHLH: Basic Helix-Loop-Helix; C4H: Cinnamate 4-hydroxylase; CHI: Chalcone isomerase; CHS: Chalcone synthase; DEGs: Differentially expressed genes; DFR: Dihydroflavonol 4-reductase; DHK: Dihydrokaempferol; EBGs: Early Biosynthetic Genes; F3'5'H: Flavonoid 3'5'-hydroxylase; F3'H: Flavonoid 3'hydroxylase; F3H: Flavanone 3-hydroxylase; FPKM: The expected number of Fragments per Kilobase of transcript sequence per Millions base pairs sequenced; GO: Gene Ontology; GSTs: Glutathione S-transferases; KEGG: Kyoto Encyclopedia of Genes and Genomes; LBD: Lateral Organ Boundaries Domain; LBGs: Late Biosynthetic Genes; OMT: Omethyltransferase; PAL: Phenylalanine ammonia-lyase; RT-qPCR: Real-time quantitative PCR; TT8: Transparent TESTA8; UFGTs: The UDP-glucose: flavonoid-3-O-glucosyltransferase

\section{Acknowledgements}

We thank Dr. Gongjun Shi and Dr. Yaowei Zhang for their kindly revisions and suggestions.

\section{Funding}

This study was financially supported by National Natural Science Foundation of China (31401895) and 'Young Talents' project of Northeast Agricultural University of China (14Q10).

\section{Availability of data and materials}

The raw RNA-Seq data for the two varieties in three replicates are available in the NCBI Sequence Read Archive (SRA) repository via accession number PRJNA359160.

\section{Authors' contributions}

LZ and YFY designed the experiments; LZ and BX performed the RNA-seq and data analysis; TW and LXF performed GRT-PCR measurement and reviewed the manuscript; MXW and JXS conducted the measurement of anthocyanin content; LZ and BX wrote the manuscript and approved the final manuscript. All authors read and approved the final manuscript.

\section{Competing interests}

The authors declare that they have no competing interests.

\section{Consent for publication}

Not applicable.

\section{Ethics approval and consent to participate} Not applicable.

\section{Publisher's Note}

Springer Nature remains neutral with regard to jurisdictional claims in published maps and institutional affiliations.

\section{Author details}

${ }^{1}$ College of Horticulture, Northeast Agricultural University, 59 Mucai street, 150030 Harbin, People's Republic of China. ${ }^{2}$ College of Agro-grassland Science, Nanjing Agricultural University, 1 Weigang, 210095 Nanjing, People's Republic of China. ${ }^{3}$ State Key Laboratory of Tree Genetics and Breeding, The Research Institute of Forestry, Chinese Academy of Forestry Sciences, 100091 Beijing, People's Republic of China.

Received: 7 December 2016 Accepted: 31 March 2017 Published online: 11 April 2017

\section{References}

1. Tanaka Y, Sasaki N, Ohmiya A. Biosynthesis of plant pigments: anthocyanins, betalains and carotenoids. Plant J. 2008;54(4):733-49.
2. He J, Giusti MM. Anthocyanins: Natural Colorants with Health-Promoting Properties. Food Sci Tech. 2010;1(1):163-87.

3. Landi M, Tattini M, Gould KS. Multiple functional roles of anthocyanins in plant-environment interactions. Environ Exp Bot. 2015:196:4-17.

4. Grotewold E. The genetics and biochemistry of floral pigments. Annu Rev Plant Biol. 2006;57(57):761-80.

5. Guo N, Cheng F, Wu J, Liu B, Zheng S, Liang J, Wang X. Anthocyanin biosynthetic genes in Brassica rapa. BMC Genom. 2014;15(1):1-11.

6. Shirley BW. Flavonoid biosynthesis: 'new' functions for an 'old' pathway. Trends Plant Sci. 1996;1(11):377-82.

7. Bogs J, Ebadi A, McDavid D, Robinson SP. Identification of the flavonoid hydroxylases from grapevine and their regulation during fruit development. Plant Physiol. 2006;140(1):279-91.

8. Davies KM, Schwinn KE. Transcriptional regulation of secondary metabolism. Funct Plant Biol. 2003;30(2):913-25.

9. Broun P. Transcriptional control of flavonoid biosynthesis: a complex network of conserved regulators involved in multiple aspects of differentiation in Arabidopsis. Curr Opin Plant Biol. 2005;8(3):272-9.

10. Peng T, Moriguchi T. The molecular network regulating the coloration in apple. Sci Hortic. 2013;163:1-9.

11. Zhang F, Gonzalez A, Zhao M, Payne CT, Lloyd A. A network of redundant bHLH proteins functions in all TTG1dependent pathways of Arabidopsis. Development. 1991;130(20):4859-69.

12. Park Kl, Ishikawa N, Morita Y, Choi JD, Hoshino A, lida S. A bHLH regulatory gene in the common morning glory, Ipomoea purpurea, controls anthocyanin biosynthesis in flowers, proanthocyanidin and phytomelanin pigmentation in seeds, and seed trichome formation. Plant J. 2007:49(4):641-54.

13. Feyissa DN, Lovdal T, Olsen KM, Slimestad R, Lillo C. The endogenous GL3, but not EGL3, gene is necessary for anthocyanin accumulation as induced by nitrogen depletion in Arabidopsis rosette stage leaves. Planta. 2009;230(4):747-54

14. Xie XB, Li S, Zhang RF, Zhao J, Chen YC, Zhao Q, Yao YX, You CX, Zhang XS, Hao YJ. The bHLH transcription factor MdbHLH3 promotes anthocyanin accumulation and fruit colouration in response to low temperature in apples. Plant Cell Environ. 2012;35(11):1884-97.

15. Xie Y, Tan H, Ma Z, Huang J. DELLA Proteins Promote Anthocyanin Biosynthesis via Sequestering MYBL2 and JAZ Suppressors of the MYB/ bHLH/WD40 Complex in Arabidopsis thaliana. Mol Plant. 2016;9(5):711-21.

16. Murillo G, Mehta RG. Cruciferous vegetables and cancer prevention. Nutr Cancer. 2001:41(1-2):17-28.

17. Keck AS, Finley JW. Cruciferous vegetables: cancer protective mechanisms of glucosinolate hydrolysis products and selenium. Integr Cancer Ther. 2004;3(3):5-12.

18. Lin Z, Li S, Chang D, Lin G, Li Y, Liu S, Chen M. The changes of pigments, phenolics contents and activities of polyphenol oxidase and phenylalanine ammonia-lyse in pericarp of postharvest litchi fruit. Acta Bot Sin. 1988:30(1):40-5.

19. Cheng F, Liu S, Wu J, Fang L, Sun S, Liu B, Li P, Hua W, Wang X. BRAD, the genetics and genomics database for Brassica plants. BMC Plant Biol. 2011;11(1):1-6.

20. Wang L, Feng Z, Wang X, Wang $X$, Zhang X. DEGseq: an R package for identifying differentially expressed genes from RNA-Seq data. Bioinformatics. 2010;26(1):136-8.

21. Young MD, Wakefield MJ, Smyth GK, Oshlack A. Gene ontology analysis for RNA-seq: accounting for selection bias. Genome Biol. 2010;11(2):R14.

22. Mao X, Cai T, Olyarchuk JG, Wei L. Automated genome annotation and pathway identification using the KEGG Orthology $(\mathrm{KO})$ as a controlled vocabulary. Bioinformatics. 2005;21(19):3787-93.

23. Mushtaq MA, Pan Q, Chen D, Zhang Q, Ge X, Li Z. Comparative leaves transcriptome analysis emphasizing on accumulation of anthocyanins in Brassica: molecular regulation and potential interactions with photosynthesis. Front Plant Sci. 2016;7:311.

24. Holton T, Cornish E. Genetics and biochemistry of anthocyanin biosynthesis. Plant Cell. 1995:7(7):1071-83.

25. Olsen KM, Lea US, Slimestad R, Verheul M, Lillo C. Differential expression of four Arabidopsis PAL genes, PAL1 and PAL2 have functional specialization in abiotic environmental-triggered flavonoid synthesis. J Plant Physiol. 2008; 165(14):1491-9.

26. Huang J, Gu M, Lai Z, Fan B, Shi K, Zhou YH, Yu JQ, Chen Z. Functional analysis of the Arabidopsis PAL gene family in plant growth, development, and response to environmental stress. Plant Physiol. 2010;153(4):1526-38. 
27. Ehlting J, Buttner D, Wang Q, Douglas CJ, Somssich IE, Kombrink E. Three 4coumarate: coenzyme A ligases in Arabidopsis thaliana represent two evolutionarily divergent classes in angiosperms. Plant J. 1999;19(1):9-20.

28. Petroni $\mathrm{K}$, Tonelli C. Recent advances on the regulation of anthocyanin synthesis in reproductive organs. Plant Sci. 2011;181(3):219-29.

29. Owens DK, Alerding AB, Crosby KC, Bandara AB, Westwood JH, Winkel BS. Functional analysis of a predicted flavonol synthase gene family in Arabidopsis. Plant Physiol. 2008;147(3):1046-61.

30. Yonekura-Sakakibara K, Nakayama T, Yamazaki M, Saito K. Modification and stabilization of anthocyanins. In: Gould K, Davies K, Winefield C, editors. Anthocyanins: Biosynthsis. functions and apllications. New York: Springer; 2009. p. 169-85.

31. Takos A, Jaffe F, Jacob S, Bogs J, Robinson S, Walker A. Light-induced expression of a MYB gene regulates anthocyanin biosynthesis in red apples. Plant Physiol. 2006;142(3):1216-32.

32. Hichri I, Barrieu F, Bogs J, Kappel C, Delrot S, Lauvergeat V. Recent advances in the transcriptional regulation of the flavonoid biosynthetic pathway. J Exp Bot. 2011;62(8):2465-83.

33. Zimmermann I, Heim M, Weisshaar B, Uhrig J. Comprehensive identification of Arabidopsis thaliana MYB transcription factors interacting with R/B-like bHLH proteins. Plant J. 2004;40(1):22-34.

34. Hartmann U, Sagasser M, Mehrtens F, Stracke R, Weisshaar B. Differential combinatorial interactions of cis-acting elements recognized by R2R3-MYB, BZIP, and BHLH factors control light-responsive and tissue-specific activation of phenylpropanoid biosynthesis genes. Plant Mol Biol. 2005;57(2):155-71.

35. Shi MZ, Xie DY. Biosynthesis and metabolic engineering of anthocyanins in Arabidopsis thaliana. Recent Pat Biotechnol. 2014;8(1):47-60.

36. Qi T, Song S, Ren Q, Wu D, Huang H, Chen Y, Fan M, Peng W, Ren C, Xie D. The Jasmonate-ZIM-domain proteins interact with the WD-Repeat/BHLH/MYB complexes to regulate Jasmonate-mediated anthocyanin accumulation and trichome initiation in Arabidopsis thaliana. Plant Cell. 2011;23(5):1795-814.

37. Goodman $C D$, Casati $P$, Walbot V. A multidrug resistance-associated protein involved in anthocyanin transport in Zea mays. Plant Cell. 2004;16(7):1812-26.

38. Gomez C, Terrier N, Torregrosa L, Vialet S, Fournier-Level A, Verries C, Souquet JM, Mazauric JP, Klein M, Cheynier V, Ageorges A. Grapevine MATE-type proteins act as vacuolar $\mathrm{H}+$-dependent acylated anthocyanin transporters. Plant Physiol. 2009:150(1):402-15.

39. Frank S, Keck M, Sagasser M, Niehaus K, Weisshaar B, Stracke R. Two differentially expressed MATE factor genes from apple complement the Arabidopsis transparent testa12 mutant. Plant Biol. 2011;13(1):42-50.

40. Conn S, Curtin C, Bezier A, Franco C, Zhang W. Purification, molecular cloning, and characterization of glutathione S-transferases (GSTs) from pigmented Vitis vinifera L. cell suspension cultures as putative anthocyanin transport proteins. J Exp Bot. 2008;59(13):3621-34.

41. Marrs KA, Alfenito MR, Lloyd AM, Walbot V. A glutathione S-transferase involved in vacuolar transfer encoded by the maize gene Bronze-2. Nature. 1995;375(6530):397-400.

\section{Submit your next manuscript to BioMed Central and we will help you at every step:}

- We accept pre-submission inquiries

- Our selector tool helps you to find the most relevant journal

- We provide round the clock customer support

- Convenient online submission

- Thorough peer review

- Inclusion in PubMed and all major indexing services

- Maximum visibility for your research

Submit your manuscript at www.biomedcentral.com/submit

) Biomed Central 